\title{
A parsimonious model for the proportional control valve
}

\author{
K F Elmer* and C R Gentle \\ Department of Mechanical and Manufacturing Engineering, Faculty of Engineering, The Nottingham Trent \\ University, Nottingham
}

\begin{abstract}
A generic non-linear dynamic model of a direct-acting electrohydraulic proportional solenoid valve is presented. The valve consists of two subsystems--a spool assembly and one or two unidirectional proportional solenoids. These two subsystems are modelled separately. The solenoid is modelled as a non-linear resistor-inductor combination, with inductance parameters that change with current. An innovative modelling method has been used to represent these components. The spool assembly is modelled as a mass-spring-damper system. The inertia and the damping effects of the solenoid armature are incorporated in the spool model. The model accurately and reliably predicts both the dynamic and steady state responses of the valve to voltage inputs. Simulated results are presented, which agree well with experimental results.
\end{abstract}

Keywords: electrohydraulic, proportional control valve, mathematical model, non-lincar, parsimonious model

\section{NOTATION}

c spring stiffness $(\mathrm{N} / \mathrm{m})$

d spring compressed length $(\mathrm{m})$

$d_{k} \quad$ spool and armature friction-velocity coefficient ( $\mathrm{N} \mathrm{s} / \mathrm{m}$ )

$D \quad$ spring free lengtl $(\mathrm{m})$

$F$ solenoid force (N)

$i \quad$ instantaneous value of current (A)

final value of current (A)

e.m.f. dependence on solenoid velocity (V s/m) circuit inductance $(\mathbf{H})$

spool and armature mass $(\mathrm{kg})$

circuit resistance $(\Omega)$

Laplace operator

time (s)

time constant (s)

e.m.f, duc to solenoid velocity (V)

supply voltage (V)

spool position (m)

$\psi \quad$ magnetic flux (Wb)

\section{INTRODUCTION}

Recent advances in electrohydraulic proportional control valves (EHPCVs) have improved their performance close to that of electrohydraulic servo valves, but without their limitations, such as sensitivity to fluid contamination, hysteresis and lack of fail-safe characteristics [1]. The EHPCV effects displacement of a flow control spool by use of one or two solenoids acting directly on the valve spool. Such a valve is shown in Fig. 1. It has slightly slower performance characteristics than the electrohydraulic servo valve and some nonlinearities, but modern integrated electronics reduce these effects to an acceptable minimum. The selection process for such valves is, however, still mathematically intense. One of the main difficulties that has restricted the application of EHPCVs has been the lack of a simple generic mathematical model to represent the nonlinearities that are present in such valves.

There are two published mathematical models for EHPCVs: a simple but inaccurate lumped parameter model [2], and a morc accurate but very complex lumped parameter model [3]. Other relevant approaches, like the lumped circuit and finite analysis methods, have been considered in the past $[4,5]$ but were judged too complex and specific to single valves. Furthermore, while these methods are attractive for their accuracy, they are also expensive in computer processing timc. If, however, the models were to be postulated semi-empirically, then the parameter values could be identified by means of simple
The MS was received on 16 March 2001 and was acepted after revision for puhlicution on 27 June 2001

* Corresponding author: Department of Mechanical and Manufacturing Engineering, Faculty of Engineering, The Nottingham Trent University. Burron Street, Nortingham NG1 $4 B U, U K$. 


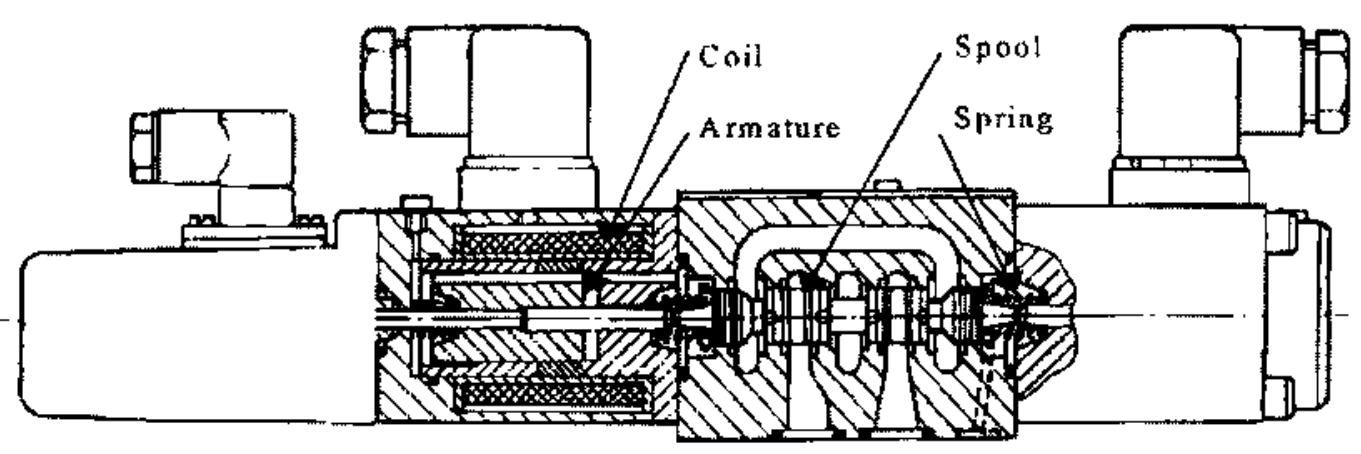

Fig. 1 An electrohydraulic proportional control valve

numerical methods using measured characteristic curves. The form of the model would then be independent of the most common differences in construction of the modelled valves.

It was therefore concluded that a semi-empirical approach could provide the basis for a new, economical, general analysis method. The method would essentially rely on constructing a lumped parameter model from theory and then assigning values to each parameter based on measurements and experimental results. The model, however, should include some further detail such as the effects of temperature change due to drive current heating [6]. This approach would therefore provide a practical yet accurate mathematical model for use in the analysis of electrohydraulic control systems.

The devclopment of the valve model was undertaken in stages corresponding to the physical components of the valve. A proposed model was produced at each stage and evaluated using the MATLAB ${ }^{\text {P }}$ analysis package. The evaluation data were then compared with those from experimental step-response tests carried out on the actual valves. The structure and parameters of the model were altered through an iterative process to achieve an accurate match.

The significant components of the valve are the solenoid and the spool. The solenoid is modelled as a firstorder system with resistance that changes with temperature owing to heating by the operating current, and inductance that reduces with increasing current value. This change happens because, as the current is increased, the magnetic flux is drawn into the magnetic circuit and therefore does not link with the currentcarrying coil windings. The force output from the solenoid operates on the spool which is a mass-springdamper with pre-loaded centring springs and a physical end stop. Movement of the spool, as measured by a linear variable differential transformer, affects two fluidflow throttle areas such that the area change is designed to be approximately linear with spool displacement.

The methods described have been applied to three different valves. These are identified in the Appendix.

\section{SOLENOID MODEL}

A proportional solenoid is very similar in operation to a conventional on/off d.c. solenoid of wet armature design as used in solenoid switching valves.

Passing a current through the solenoid coil creates a magnetic force that pulls the moving armature towards a pole piece, the magnitude of the force being proportional to the coil current. The solenoid force is transmitted to the valve spool by means of a push pin. The main difference between a proportional solenoid and a simple on-off solenoid is in the design of the armature, pole piece and core tube assembly. These are shaped to give a more constant force over the working range of solenoid stroke so that the force produced is proportional to coil current, independent of armaturc position.

The simplest form of solenoid model is a resistor in series with a linear inductor [2] as shown in Fig. 2. The voltage-current relationship can be easily derived by equating the voltages in the circuit:

$$
V=V_{\mathrm{R}}+V_{\mathrm{L}}=i R+L \frac{\mathrm{d} i}{\mathrm{~d} t}
$$

There is no need to model the magnetic characteristics of the solenoid assembly in this case since the flux produced by the solenoid is effectively proportional to the coil current, independent of armature position. The output force, $F$, that is generated is in turn directly proportional to the solenoid flux:

$$
F \propto \psi \propto I
$$

\subsection{Variable inductance}

When a coil is wound on a closed magnetic circuit the problem of defining the inductance has to be considered because, as observed by Hughes [7], the flux linking the coil windings is not directly proportional to the current in the coil. As the value of an (alternating) current through such a coil is increased from zero, the value of the inductance increases to a maximum and then 


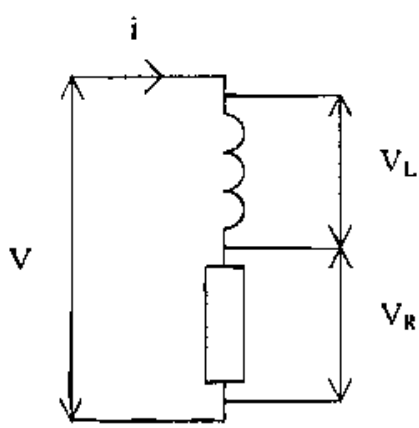

a) Circuit Diagram

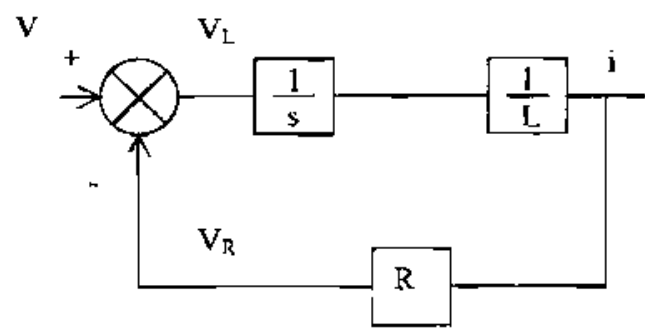

b) Block Diagram

Fig. 2 Linear solenoid model

decreases. This implies that as the d.c. current increases the magnetic flux is drawn into the low-reluctance magnetic circuit, and therefore does not link with the current-carrying coil windings. The true value of the coil inductance then varies with the instantancous value of the current in the coil, as shown in the modified form of equation (1):

$$
V=V_{\mathrm{R}}+V_{\mathrm{I}}=i R+L_{(i)} \frac{\mathrm{d} i}{\mathrm{~d} t}
$$

Solving for $L_{(i)}$ yields

$$
L_{(i)}=\frac{V-i R}{\mathrm{~d} i / \mathrm{d} t}
$$

For a step input, $V=$ constant.

Thus, at any $i, \mathrm{~d} i / \mathrm{d} t$ can be measured and a value of $L_{(i)}$ can be evaluated as a function of $i$. For the currenttime data shown in Fig. $3 \mathrm{~b}$, the inductance is found empirically to be as shown in Fig. 3c. This result, the shape of the resultant inductance-current curve in Fig. $3 \mathrm{c}$, agrees with that predicted by Hughes [7].

If the reciprocal of the inductance is plotted as a function of the current, the result is a straight line as shown in Fig. 3d. Thus

$$
\frac{1}{L_{(i)}}=a i+b
$$

or

$$
\frac{\mathrm{d} i}{\mathrm{~d} t}=\frac{V-i R}{L_{(i)}}=(a i+b)(V-i R)
$$

This relationship is significant because the behaviour can now be readily simulated on MATLAB.

Figure 4 shows the block diagram model with and without modifications to describe the effect. There are two loops in the modified model, the lower loop representing the circuit resistance as in a linear inductor. The upper loop represents the reciprocal inductance, increasing from a minimum value as the circuit current increases. The values shown are measurements taken directly from one of the valves under test, with the cir- cuit resistance measured at $3.05 \Omega$ for the temperature of the tests shown and the intercept and slope of the curve in Fig. 3d providing the zero current (b) and gain (a) values for the inductance loop respectively, with reference to equation (5) variables.

\subsection{Velocity limit}

The input voltage to the solenoid is also opposed by a spool-velocity-dependent term, as the velocity of the spool gencrates opposition to the moving force. This can be simulated in MATLAB by representing voltage to the inductor as $V-i R-k_{v} \mathrm{~d} x / \mathrm{d} t$. The effect is discussed below with the spool model because it is measured along with the spool dynamics.

\subsection{Variable resistance}

In most applications, EHPCVs give excellent reliability and consistency of performance. However, in some applications where such a valve is held open for a long
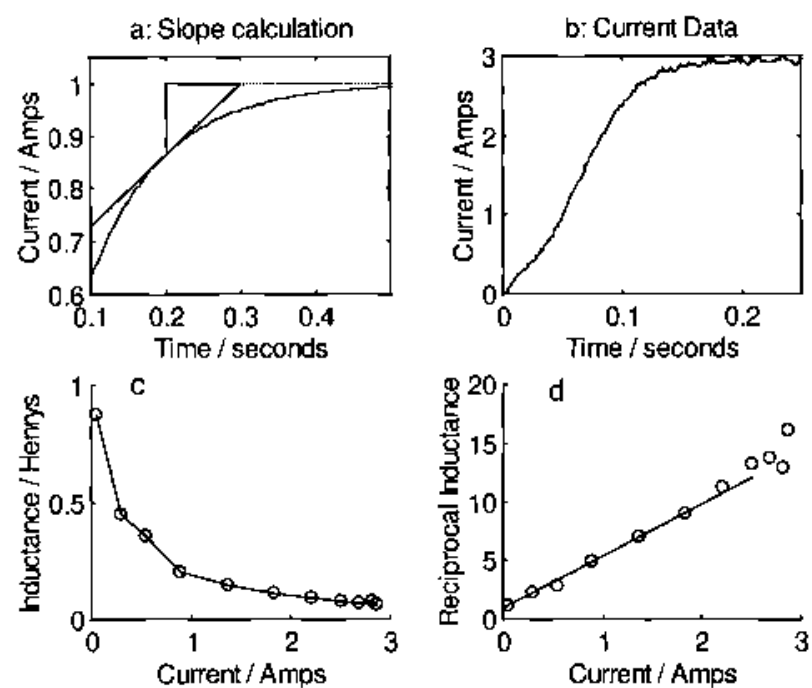

Fig. 3 Inductance-current relationship 

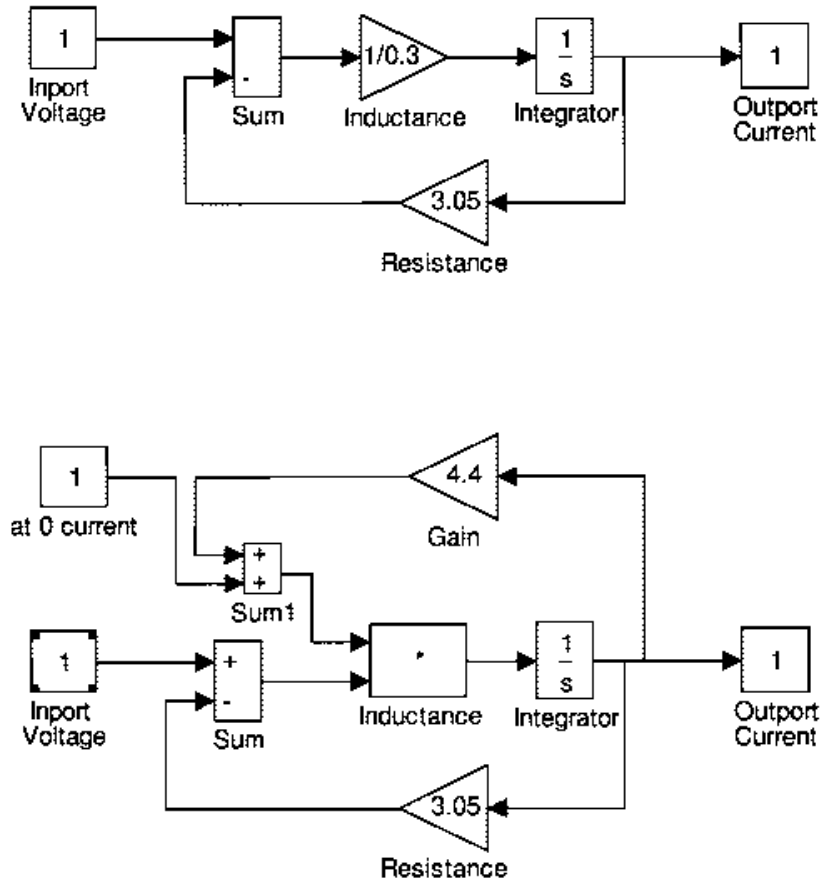

Fig. 4 Non-lincar solenoid model

period of time, the dynamic performance of the valve can be affected by a large change of coil resistance owing to heating of the solenoid by the operating current [6].

In order to provide a complete description of the solenoid response over a range of coil operating temperatures, the resistance value $R$ in the final solenoid model was computed from the coil temperature of each test using the temperature coefficient of resistance of the coil material. All of the test results described in this paper were from tests carried out with the solenoid coil at $50^{\circ} \mathrm{C}$.

\subsection{Experimental results}

Once the structure of the solenoid model had been determined, the simulation process reduced to identifying suitable values for the constants in the blocks.

In order to observe temperature effects, all experiments were carried out at known temperatures within the normal operating range for the valves. The solenoid under test was heated to the required temperature prior to each experiment by passing a constant current through the coil.

The solenoid was tested by locking the armature in position against a piezoelectric force transducer and applying a voltage input to the solenoid coil. A digital storage oscilloscope was used to sample and store the voltage, current and foree data generated by the solenoid.
Force output tests with currents rising up to the maximum operating value were carried out for 18 different armature positions over the $3 \mathrm{~mm}$ stroke length. One of the resulting three-dimensional plots is shown in Fig. 5. This plot confirms that the force generated by the solenoid is proportional to the coil current and does not change significantly over the normal stroke operating range of the valve.

Step voltage input tests were carried out with the armature locked at $1 \mathrm{~mm}$ displacement using different slep voltages in order to confirm that the modet was valid with different finat current values. The results of these tests are shown in Fig. 6. Also shown in this figure are the simulation results obtained using the solenoid model, both with fixed inductance and changing inductance. In the plots shown, experimental data are represented as solid lines, and simulated data as broken or dashed lines. The d.c. step voltage used for each test was sciected to give the final current achieved in the resistance of the solenoid. The parameter values used in the final model were identified by curve-fitting methods from these results as described above. Figure 6 demon-

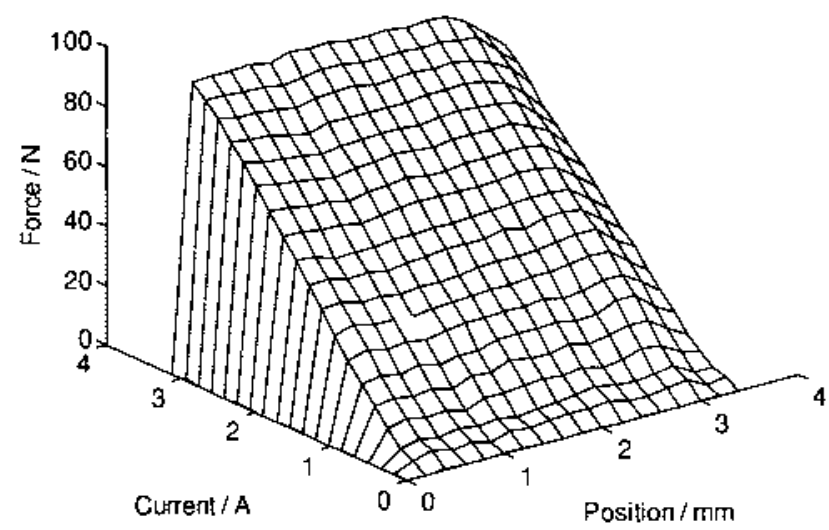

Fig. 5 Three-dimensional plot of solenoid force outpul

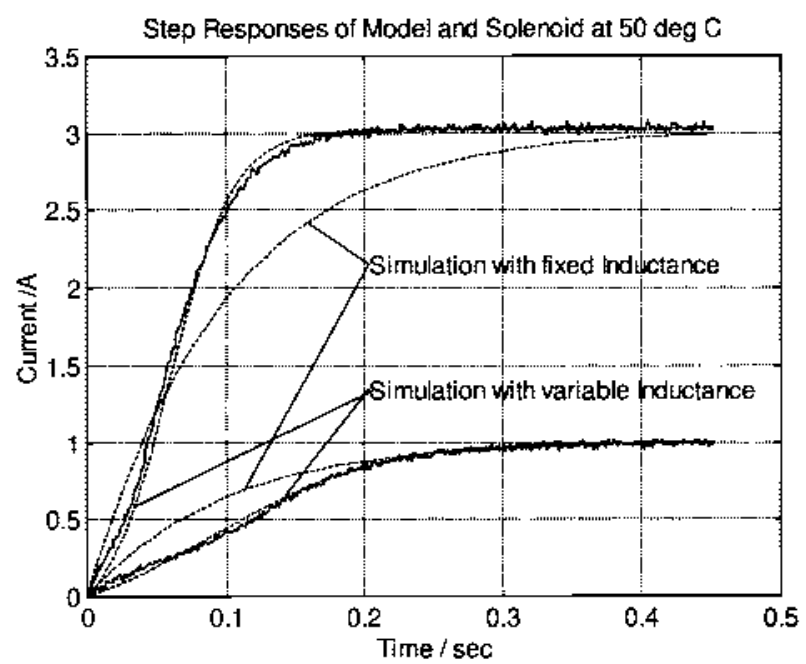

Fig. 6 Force step response of modet and solenoid 
strates the large difference between the fixed inductance model and the variable inductance model. There is a maximum opposition to changing current at small current values and minimum opposition at large current values, leading to an $S$-shaped current curve instead of the classic exponential current increase.

\section{SPOOL MODEL}

The armature and spool arrangement was modelled as a lumped parameter mass-spring-damper system [2], as shown in Fig. 7. The parameter values for the model components were obtained in two ways:

(a) by direct measurement and calculation from the valve component dimensions;

(b) from experiments carried out on the valves to measure spool position and velocity response to a voltage step input.

The input force provided by the solenoid to move the spool is represented as $F$. There were two outputs from the model: $x$ is the spool position output, represented as a signal proportional to the spool position in the valve body, and $v_{0}$ is a voltage signal, which is proportional to the velocity of the spool movement because the spool velocity opposes the flux that provides the moving force. This effectively limits the velocity of the spool. Aperture metering between the valve spool and body is designed to permit oil flow through the valve in proportion to the spool displacement. Flow reaction forces are neglected.

The position output signal is

$$
\frac{x}{F}=\frac{1 / m}{s^{2}+\left(d_{k} / m\right) s+(c / m)}
$$

The spool mass, the spring stifiness and the friction velocity coefficient were provided as simple constants, measured and calculated directly from the components. The solenoid e.m.f. generated by the velocity of the spool was estimated and adjusted to provide an accurate simulation of the complete valve response.

This velocity feedback signal is

$$
\frac{v_{0}}{F}=\frac{(1 / m) k_{\iota} s}{s^{2}+\left(d_{k} / m\right) s+c / m}
$$

where $k_{l}$ is the c.m.f. sensitivity of the solenoid to the velocity of the spool.

From these formulac, the model shown in Fig. 7 was produced for the valve spool. The velocity feedback signal is shown on the left-hand side of the diagram and the position output signal, $x$, is shown on the right-hand side. During the final testing of the model, it was found that an additional transport delay of a few milliseconds was also needed in this $v_{0}$ signal path to achieve the correct frequency of oscillation in the spool velocity.

When the valve solenoids are de-energized the valve spool is centred by force from two opposing helical compression springs. These springs act against collars set against the first step from the end of the spool, as shown in Fig. 1. However, when the spool is fully centred, the collars stop against a step in the valve body. When in this condition, the springs are both held in compression against the valve body to a length $d_{\text {, which }}$ is shorter than their free length $D$. This means that as soon as one of the solenoids of the valve is energized it acts against a pre-load of $(D-d) c \mathrm{~N}$.

The valve model was therefore modified to incorporate this spring pre-load by the use of the SIMULINK SIGN and GAIN blocks, as shown in Fig. 8 to provide opposition to cither direction of spool displacement. The effect of the use of these blocks is that any non-zero spool displacement signal generates a force signal of the opposite polarity, equal to the actual spring pre-load.

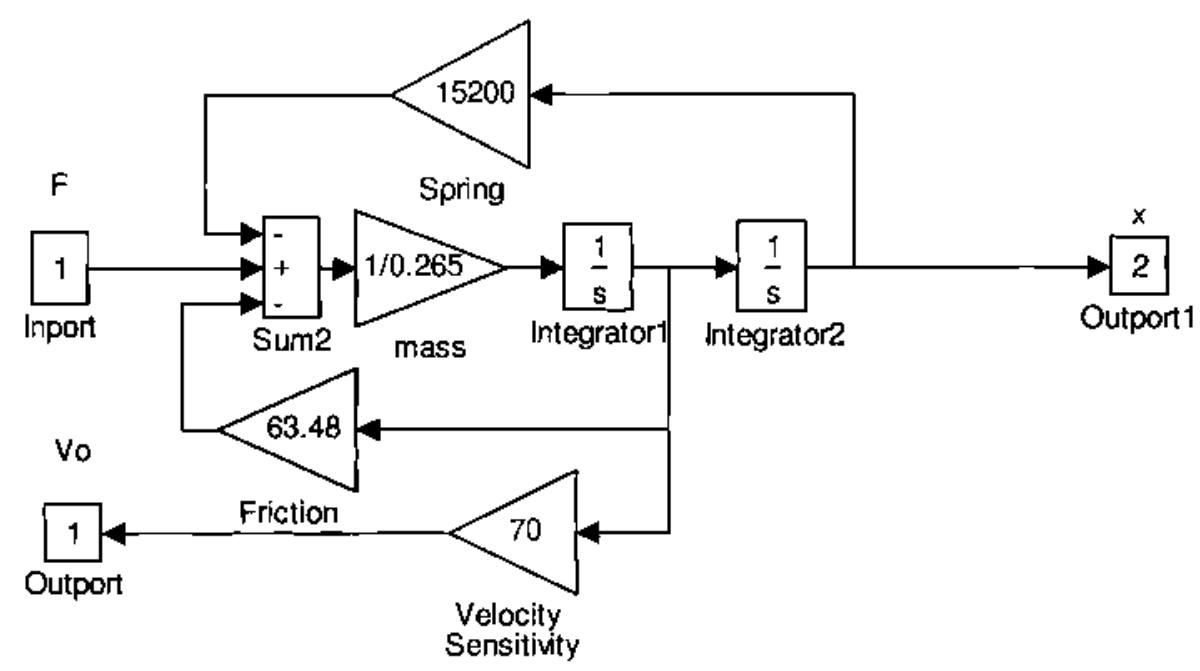

Fig. 7 Spool model 
To bring the model spool to a halt at the effective end of its range of movement, an additional feedback loop was used, as shown in Fig. 8. This made use of DEAD ZONE and GAIN blocks to effectively provide an equal and opposite force to prevent movement beyond the range of spool travel. The range is $3 \mathrm{~mm}$ in either direction for the valves considered in this study. With the DEAD ZONE block set to $3 \mathrm{~mm}$, any increase in the spool displacement signal beyond this value generates a very large feedback signal with the opposite polarity to that of the force input to the spool model. This signal will then increase until equilibrium is reached such that both spool movement and the acceleration integrator are zeroed.

\subsection{Experimental results}

Parameter values for the model components were obtained by direct measurement and calculation except for the sensitivity of the solenoid e.m.f. to spool velocity, which was selected to obtain an accurate simulation of the complete valve response.

A valve was step-response tested to measure the change of spool displacement and velocity with time. The velocity of the solenoid spool was measured during the tests using a velocity transducer secured in a special clamp mounted on the end of the valve body in place of the second solenoid. A digital storage oscilloscope was used to gather the data. The spool position and velocity outputs for a zero current to $3 \mathrm{~A}$ step-voltage input test are shown in Fig. 9, together with simulation results. In the plots shown, experimental data are represented as solid lines, and simulated data as broken or dashed lines. The d.c. step voltage used in this case was $9.14 \mathrm{~V}$, selected to give a final current of $3 \mathrm{~A}$ in the resistance of the solenoid at that temperature. In order to fine-tune the model, a transport delay of just $2 \mathrm{~ms}$ was added into the spool velocity feedback signal so that the oscillation in spool velocity acted at the correct frequency. This transport delay is shown in Fig. 8. Note that this is not the same as the delay used by Matten [2] to represent the large opposition to current increase from zero.

The simulation with fixed inductance value shows a response in which the solenoid force output exceds the spring pre-load too early, causing a clearly visible error. A delay is required; in the model produced by Matten [2], this delay was simulated by the use of a simple, variable $(10 \mathrm{~ms})$ dead time in the solenoid, which is 'longest if the solenoid is previously not energised'. The simulation with variable inductance value shows that the predicted spool position in response to the applied step voltage stays within 2 per cent of the actual value. This compares favourably with the maximum step-response position crrors of the models published by Matten [2] at 3.7 per cent and Gamble and Vaughan [3] at 4.76 per cent, both for similar valves where the same model is applicable. The accuracy of the velocity response for variable inductance is rather worse at about $10-15$ per cent. Spool velocity data are not presented in these other papers, so only the spool position traces can be compared for accuracy.

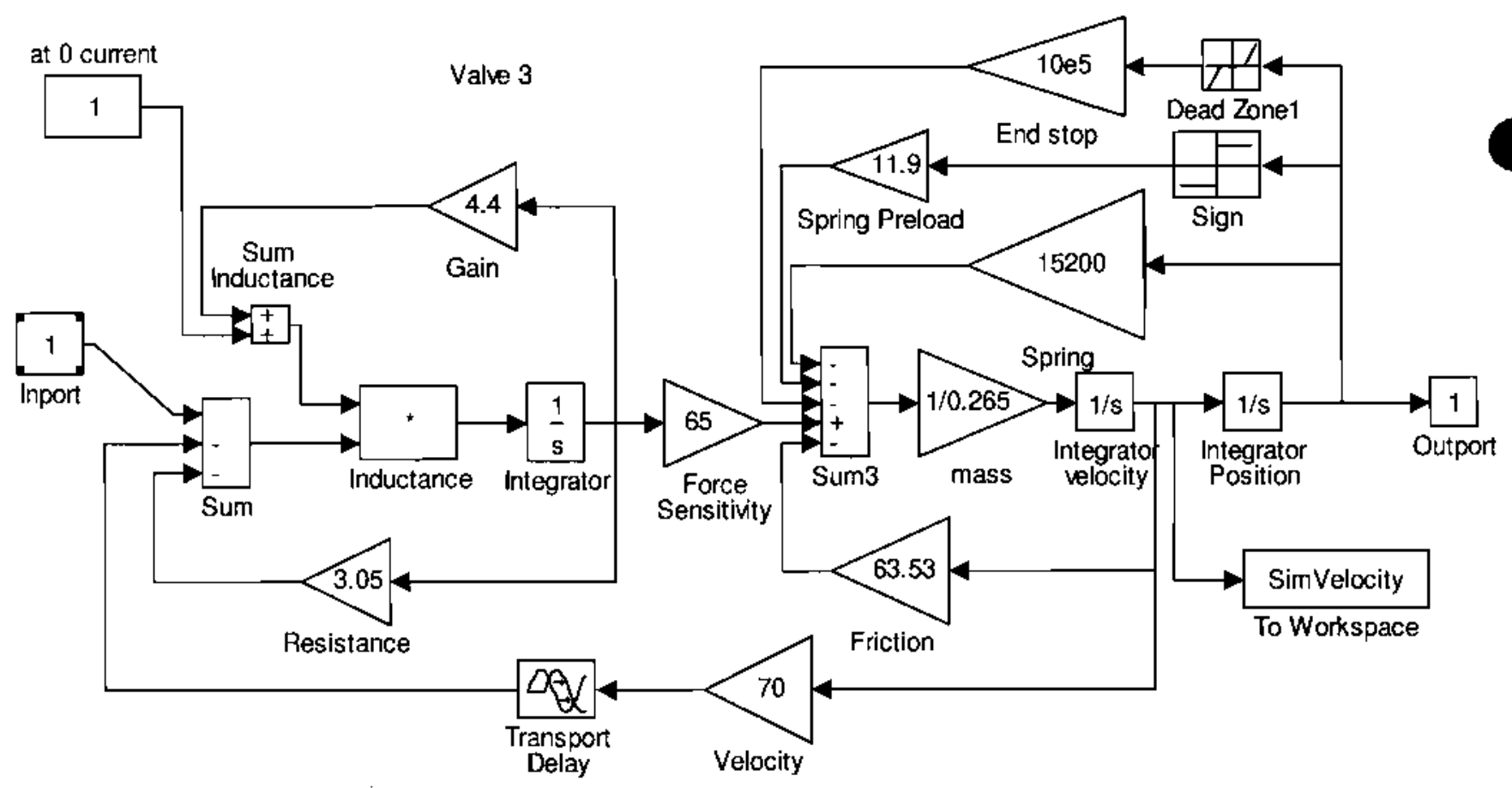

Fig. 8 Complete valve model 


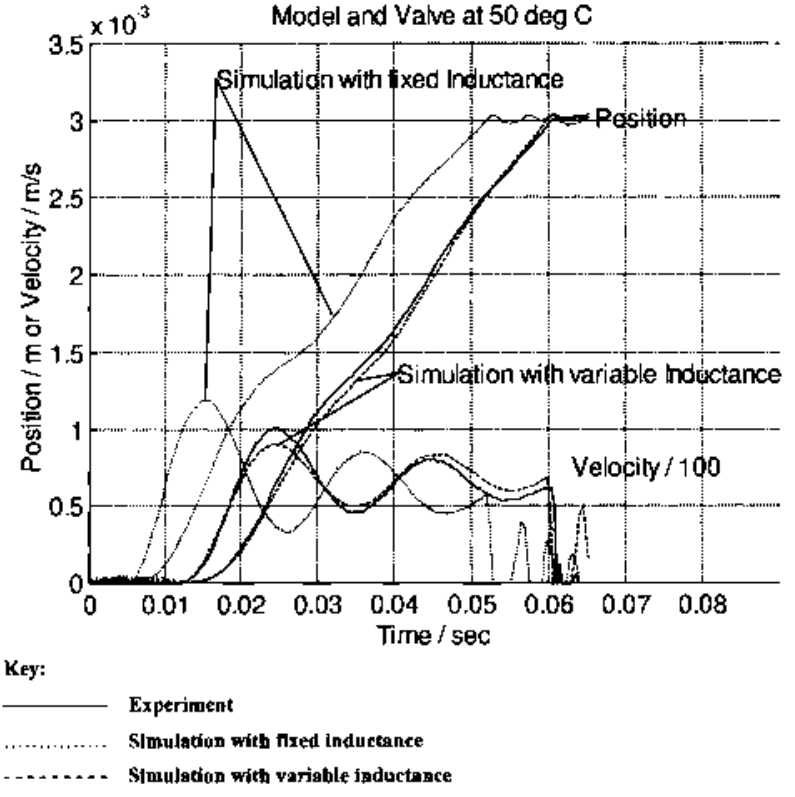

Fig. 9 Valve step-response results

It is interesting to note that the simpler of these two models does appear to give a better accuracy than the complex one, but this is only true for the case where there is zero current in the solenoid before the step input is applied.

\section{CONCLUSIONS}

A generic, parsimonious dynamic simulation model has been produced that accurately predicts the spool displacement of a proportional solenoid valve to voltage input. In addition, the model simulates the effects of variation of solenoid inductance with current and the variation of solenoid resistance with temperature owing to current heating.

This mathematical model is more detailed than the simple model of Matten [2] but simpler than the accurate but complex model of Gamble and Vaughan [3]. The accuracy of the model in predicting spool position was confirmed to be better than 98 per cent by comparison of actual and simulated step-response and frequencyresponse tests.

Because the model is completely general and structured in a straightforward manner, it can be relatively easily reproduced and modified to take account of changes in valve size and design. Owing to its simplicity, it can be used to simulate the performance of the valve in a complex hydraulic control system. Other similar valves can be modelled by using the same model structure with new parameter values obtained by a combination of direct component measurement and curve fitting from valve test data.

\section{ACKNOWLEDGEMENT}

The authors would like to express their thanks to Vickers Systems Limited for their assistance in this project.

\section{REFERENCES}

1 Cullman, J. Electrohydraulic motion control. Automation, May $1990,37,4446$.

2 Matten, N. Measures to significantly improve the dynamics of proportional directional valves. In Proceedings of the Third Scandinavian International Conference on Fluid Power. 1994. Vol. 2, pp. 177-197.

3 Gamble, J. B. and Vaughan, N. D. The modelling and simulation of a proportional solenoid valve. Trans. ASME, J. Dyn. Syst, Measmt Control, 1996, 118(1), 120125.

4 Lequesne, B. Finite element analysis of a constant force solenoid for fluid flow control. In Proceedings of the IEEE Industrial Applications Society Meeting, 1987, pp. 46-51.

5 Jelali, M. and Schwarz, H. Nonlinear identification of hydraulic servo-drive systems. IEEE Control Syst. Mag., $1995,15(5), 17-22$.

6 Elmer, K. F., Henthorn, K. S. and Skellern, P. Sell heating effects on the dynamics of a proportional control valve. $J$. Measmt Control, May 1998, 31(4), 101104.

7 Hughes, E. Electrical Technology, 1970 (Longman Scientific and Technical, London).

\section{APPENDIX}

The valves modelled were electrohydraulic proportional throttle valves with a feedback transducer. Technical data are given in Table 1 .

Table 1 Technical data

\begin{tabular}{llll}
\hline $\begin{array}{l}\text { Model } \\
\text { number }\end{array}$ & $\begin{array}{l}\text { ISO 4401 } \\
\text { size }\end{array}$ & $\begin{array}{l}\text { Rated } \\
\text { flow range } \\
\text { (lmin) }\end{array}$ & $\begin{array}{l}\text { Maximum } \\
\text { operating pressure } \\
\text { (bar) }\end{array}$ \\
\hline I (KFDG4V-3)* & 03 & $7-28$ & 350 \\
2 KFDG4V-3 & 03 & $7-28$ & 350 \\
3 KFDG4V-5 & 05 & 5070 & 315 \\
\hline
\end{tabular}

\footnotetext{
* Fitted with a modificd solenoid type
} 